\title{
Scaling of sensorimotor control in terrestrial mammals
}

\author{
Heather L. More ${ }^{1}$, John R. Hutchinson ${ }^{2}$, David F. Collins ${ }^{3}$, \\ Douglas J. Weber ${ }^{4}$, Steven K. H. Aung ${ }^{5}$ and J. Maxwell Donelan ${ }^{1, *}$ \\ ${ }^{1}$ Department of Biomedical Physiology and Kinesiology, Simon Fraser University, Burnaby, \\ British Columbia, Canada V5A 1 S6 \\ ${ }^{2}$ Structure and Motion Laboratory, Department of Veterinary Basic Sciences, The Royal Veterinary College, \\ London NW1 0TU, UK \\ ${ }^{3}$ Faculty of Physical Education and Recreation, Centre for Neuroscience, University of Alberta, Edmonton, \\ Alberta, Canada T6G $2 \mathrm{H} 9$ \\ ${ }^{4}$ Department of Physical Medicine and Rehabilitation, University of Pittsburgh, Pittsburgh, PA 15213, USA \\ ${ }^{5}$ Department of Medicine and Family Medicine, Faculty of Medicine and Dentistry, University of Alberta, \\ Edmonton, Alberta, Canada T6G $2 \mathrm{H} 9$
}

\begin{abstract}
Sensorimotor control is greatly affected by two factors - the time it takes for an animal to sense and respond to stimuli (responsiveness), and the ability of an animal to distinguish between sensory stimuli and generate graded muscle forces (resolution). Here, we demonstrate that anatomical limitations force a necessary trade-off between responsiveness and resolution with increases in animal size. To determine whether responsiveness is prioritized over resolution, or resolution over responsiveness, we studied how size influences the physiological mechanisms underlying sensorimotor control. Using both new electrophysiological experiments and existing data, we determined the maximum axonal conduction velocity (CV) in animals ranging in size from shrews to elephants. Over the 100-fold increase in leg length, CV was nearly constant, increasing proportionally with mass to the 0.04 power. As a consequence, larger animals are burdened with relatively long physiological delays, which may have broad implications for their behaviour, ecology and evolution, including constraining agility and requiring prediction to help control movements.
\end{abstract}

Keywords: scaling; conduction velocity; nerve; mammal; locomotion; sensorimotor

\section{INTRODUCTION}

Terrestrial mammals span a wide range of sizes, with the largest elephant being several million times more massive than the smallest shrew. As a consequence of their size, large and small animals experience very different physical challenges. If an elephant falls, for example, it risks grave injury, while a similar tumble for a shrew would probably be inconsequential (McMahon \& Bonner 1983). To begin to understand how small and large animals coordinate their movement in the face of differing physical challenges like these, one must first determine how size influences the physiological mechanisms underlying sensorimotor control.

Sensorimotor control is greatly affected by two factors-the time it takes for an animal to sense and respond to stimuli, and the ability of an animal to distinguish between sensory stimuli and generate graded muscle forces (Enoka 1995). The first factor, which we refer to as responsiveness, encompasses many sources of delay. Even in the simple monosynaptic stretch reflex, a response only occurs after delays from sensing, conduction, synaptic transmission and force generation. If all

\footnotetext{
* Author for correspondence (mdonelan@sfu.ca).
}

Electronic supplementary material is available at http://dx.doi.org/10. 1098/rspb.2010.0898 or via http://rspb.royalsocietypublishing.org. other sources of delay are constant, responsiveness is proportional to axonal conduction velocity (CV), which is in turn directly proportional to axon diameter (Gasser \& Grundfest 1939; Hursh 1939; Rushton 1951; Boyd \& Kalu 1979; Arbuthnott et al. 1980; Hoffmeister et al. 1991) (figure 1a). The second factor, which we refer to as resolution, is determined by the number of sensory receptors and motor units that an animal possesses and is proportional to the number of axons that innervate a unit volume. Just as the size of an electrical cable depends on the number and gauge of wires inside, a nerve's crosssectional area is dependent on the number and diameter of its axons. A nerve of constant cross-sectional area must decrease its average axon diameter, and thus decrease responsiveness, in order to increase its resolution by increasing the number of axons.

As animal size increases, the trade-off between responsiveness and resolution becomes more acute. Most terrestrial mammals are approximately geometrically similar-linear dimensions such as leg length increase by a constant factor, areas such as nerve cross-sectional area increase proportional to the square of linear dimensions, and volumes as well as mass increase proportional to the cube of linear dimensions (Alexander 1979). To maintain responsiveness by keeping axonal conduction delay constant, axonal $\mathrm{CV}$ and therefore diameter $d$ must also increase in a geometrically similar manner. That is, $\mathrm{CV}$ 
(a) responsiveness and resolution definitions

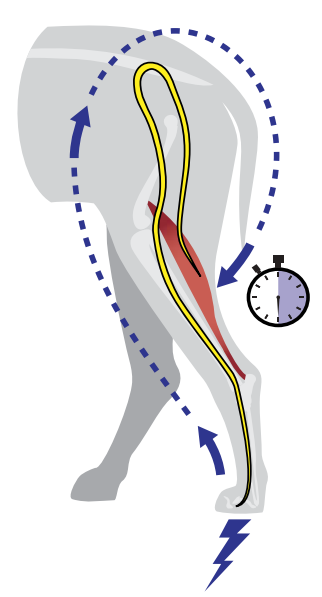

(i)

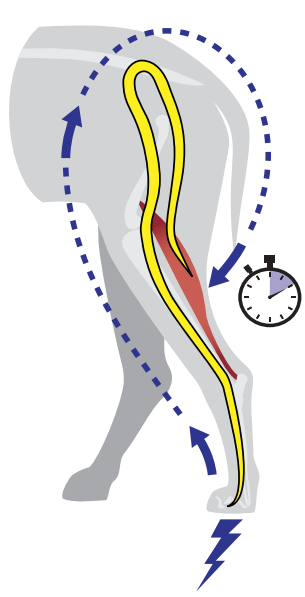

high

responsiveness

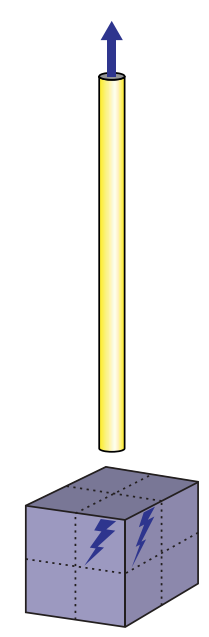

(ii) low

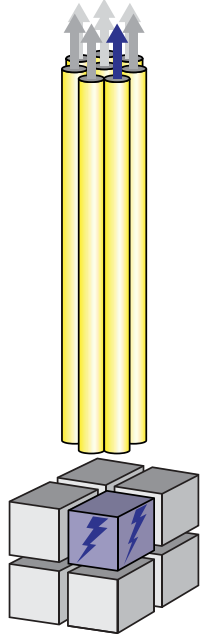

high

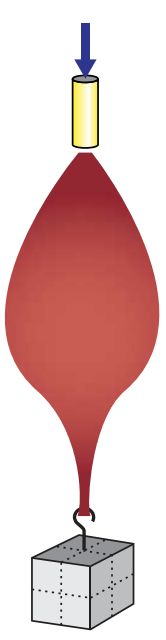

(iii) low

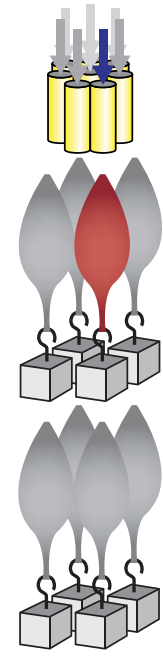

high

sensory resolution

(b) responsiveness and resolution tradeoff

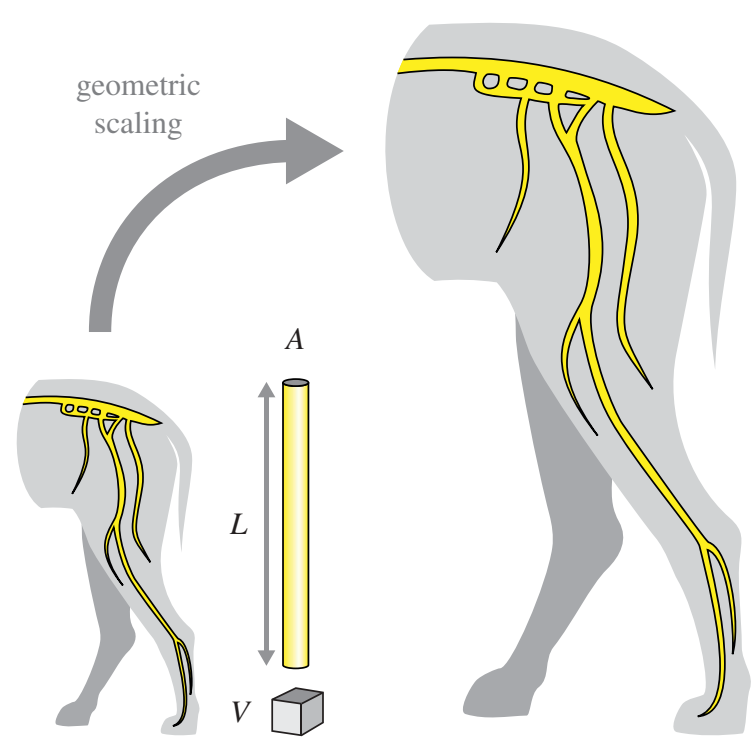

$4 A$

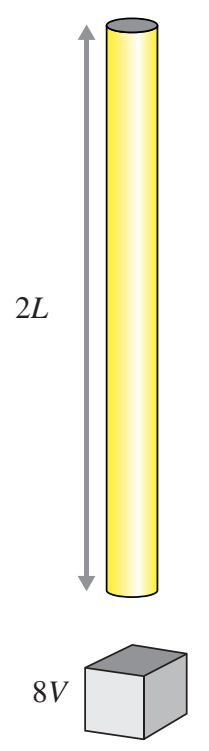

equal

responsiveness

$8 A$

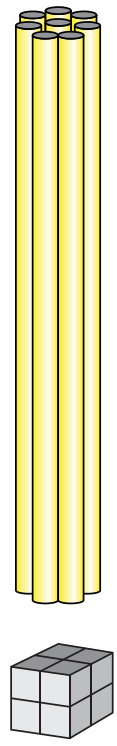

equal resolution

Figure 1. Theoretical scaling of axons and nerves. (a) Responsiveness (i) refers to the speed at which an animal can sense and respond to stimuli. If all other factors are constant, pathways with larger diameter axons have higher responsiveness. Resolution refers to the ability of an animal to accurately distinguish stimuli (ii) and generate graded muscle forces (iii). More axons innervating a given volume of tissue gives a higher resolution. (b) Possible nerve structures resulting from increased animal size. The conceptual animal body is shown as a grey cube, with a simplified nerve containing one axon represented by the yellow cylinder. As animal size doubles, geometric scaling predicts that nerve length doubles from $L$ to $2 L$, total nerve cross-sectional area increases from $A$ to $4 A$ and tissue volume increases from $V$ to $8 V$. This maintains responsiveness, but results in decreased resolution. If the large animal were to have axons of the same cross-sectional area as the small animal and maintain an equal resolution, with each axon innervating the same tissue volume as in the small animal, it would need 8 axons and have a total cross-sectional area of $8 A$. If the large animal were to maintain both responsiveness and resolution, it would need 8 axons, each with a cross-sectional area of $4 A$, giving a total nerve cross-sectional area of $32 A$ (right). Maintaining both responsiveness and resolution over large increases in animal size would require impossibly large increases in nerve crosssectional area.

and diameter would be directly proportional to leg length. To maintain resolution, the number $N$ of axons innervating a given volume of tissue must remain constant, requiring the number of axons to increase proportional to mass and with the cube of leg length. Therefore, to maintain both responsiveness and resolution, total nerve crosssectional area $A$ would need to increase proportional to the fifth power of leg length $L$ (figure $1 b$ ):

$A \propto N d^{2} \Rightarrow A \propto L^{3} L^{2} \Rightarrow A \propto L^{5}$. 
To have the same absolute responsiveness and resolution as a shrew with a leg length of $3 \mathrm{~cm}$ and a sciatic nerve $0.3 \mathrm{~mm}$ in diameter, an elephant with a leg length of $3 \mathrm{~m}$ would require a sciatic nerve with the impossible diameter of $30 \mathrm{~m}$.

The trade-off between responsiveness and resolution would be ameliorated, but not solved, if the number of required sensory receptors scaled with animal surface area rather than volume, as is probable for some sensory receptors such as cutaneous receptors (Matthews 1972). The trade-off would also be lessened if responsiveness depended not on absolute time but on the relative time required to respond. For example, both the time required to fall to the ground and the stride period at equivalent speeds increase with the square root of leg length (Heglund et al. 1974; Alexander 2002). Even if both these scenarios occurred, total nerve cross-sectional area would still need to increase proportional to the cube of leg length, which is faster than predicted by geometric scaling and would still result in an insupportable increase in nerve diameter over a six order of magnitude increase in size. Because different-sized animals often have to respond to the same stimuli from the external world, it may be important under some circumstances for them to have the same absolute performance in their sensorimotor control. For example, a disease-infected insect may be a threat to both shrews and elephants. If it were to land on either animal, the animal must be able to detect the insect's position accurately (resolution) and swat the threat away before it gets bitten (responsiveness).

While an increasing tension between responsiveness and resolution seems inevitable with increases in size, an intriguing question is whether responsiveness is prioritized over resolution, resolution over responsiveness, or both are compromised. We sought an answer by investigating the previously unknown relationship between maximum axonal $\mathrm{CV}$, axon diameter and body size in terrestrial mammals.

\section{MATERIAL AND METHODS}

All procedures were approved by the SFU Animal Care Committee. Owing to our extensive methods, we present here a summary of our electrophysiology, systematic review and histology protocols-detailed descriptions are provided in the electronic supplementary material.

\section{(a) Shrew electrophysiology}

We acquired data from six least shrews (Cryptotis parva). Each shrew was anaesthetized with isoflurane and their breathing and temperature were monitored throughout the experiment. We recorded surface electromyography (EMG) activity from the medial gastrocnemius while evoking reflexes in this muscle using electrical stimulation of the sciatic nerve (figure $2 a$ ). First, we exposed the sciatic nerve and medial gastrocnemius, then placed two monopolar stimulating electrodes as far apart as possible on the surface of the sciatic nerve and a bipolar EMG recording electrode on the surface of the medial gastrocnemius. We delivered 30 electrical impulses to the surface of the sciatic nerve at each location and measured a distance of $5 \mathrm{~mm}$ between the two stimulating electrodes. The shrew was euthanized when all recordings were complete. For each shrew, we averaged the EMG responses and measured the time between the onset of stimulation artefact and the onset of muscle activity at each of the two stimulation sites. We then divided the distance between stimulation sites by the difference in latency between the sites to give $\mathrm{CV}$.

\section{(b) Elephant electrophysiology}

We acquired data from one Asian elephant (Elephas maximus) while the animal was standing and eating under the care of the handlers. We recorded surface EMG activity from the medial gastrocnemius while evoking reflexes in this muscle using two techniques: electrical stimulation of the tibial nerve and percussion of the Achilles tendon (figure $2 b$ ). We delivered electrical impulses to the tibial nerve using stimulating electrodes consisting of two thin wires inserted approximately $8 \mathrm{~cm}$ deep at the popliteal fossa. Over a period of 2-3 minutes, we delivered 13 stimuli at an intensity which evoked a Hoffman reflex in the medial gastrocnemius (Misiaszek 2003). Next, we delivered mechanical impulses to the tibial nerve using a standard Taylor percussion reflex hammer to percuss the Achilles tendon $(n=15)$ at a strength that was sufficient to evoke a reflexive muscle twitch. We measured a distance of $0.56 \mathrm{~m}$ between the tendon tap and electrical stimulation sites. $\mathrm{CV}$ was then found in the same way as for the shrew.

\section{(c) CV systematic review}

We searched the literature for maximum axonal $\mathrm{CV}$ values measured for a wide range of animal masses (table S1 in the electronic supplementary material). We used data from 23 independent studies reporting $\mathrm{CV}$ values for only one to three species each. To ensure as accurate a representation of $\mathrm{CV}$ as possible, we included a variety of experimental protocols as well as both motor and sensory values where possible. A one-way ANOVA found that there was no evidence of consistent variation in $\mathrm{CV}$ based on whether it was motor or sensory $(p=0.8)$, therefore we continued to consider both types of $\mathrm{CV}$ in our analysis. Some studies did not report masses of their animals-we estimated these masses from the literature. Least-squares linear regression of logarithmically transformed data determined the exponent of this relationship. A phylogenetically independent contrasts analysis indicated no significant phylogenetic signal in our data; therefore, we performed this regression without adjusting for evolutionary history.

\section{(d) Nerve fixation and imaging}

We obtained one shrew sciatic nerve sample from an anaesthetized animal whose sciatic nerve had been exposed as for the shrew CV experiments, and one elephant sciatic nerve sample from an adult African elephant cadaveric hindlimb (Miller et al. 2008). Immediately after each sample was obtained, we immersed it in 4 per cent paraformaldehyde and 1 per cent glutaraldehyde in $0.1 \mathrm{M}$ phosphate buffer $(\mathrm{pH} 7.4$ ) and stored it at $4{ }^{\circ} \mathrm{C}$ until further processing. We processed the fixed shrew and elephant nerves in the same manner. First, we stained the nerves with osmium tetroxide, then dehydrated them in ascending grades of ethanol. We embedded the dehydrated nerves in plastic, polished transverse sections of each nerve and secured the nerves to stubs that were then coated with carbon in preparation for scanning electron microscopy. A scanning electron microscope imaged the nerves at a magnification of approximately $1500 \times$. It was necessary for us to take multiple images to cover the entire cross section of one nerve-the images were stitched together using a custom-written MATLAB 
(a) shrew CV experiment
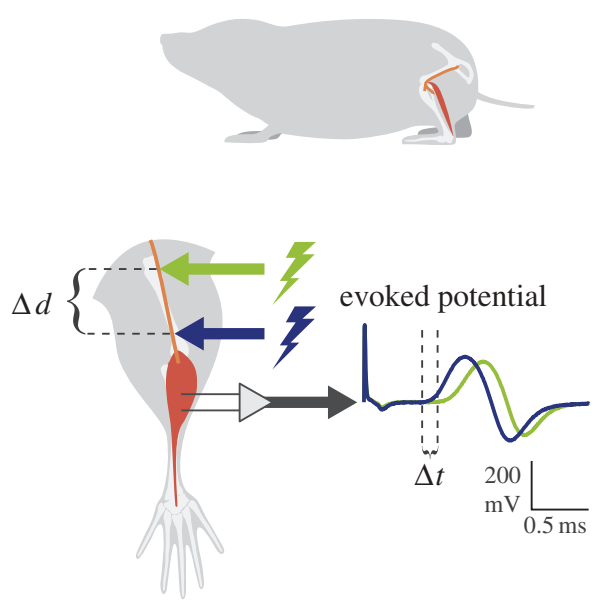

(b) elephant CV experiment

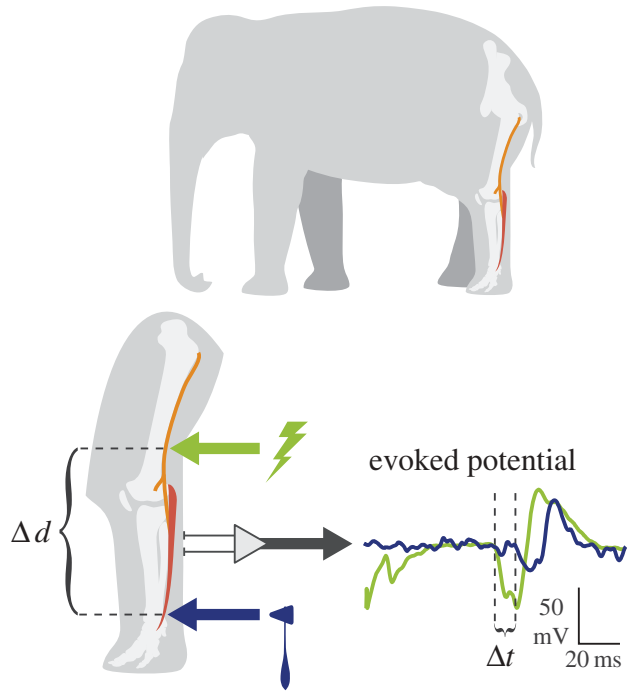

(c) scaling of conduction velocity

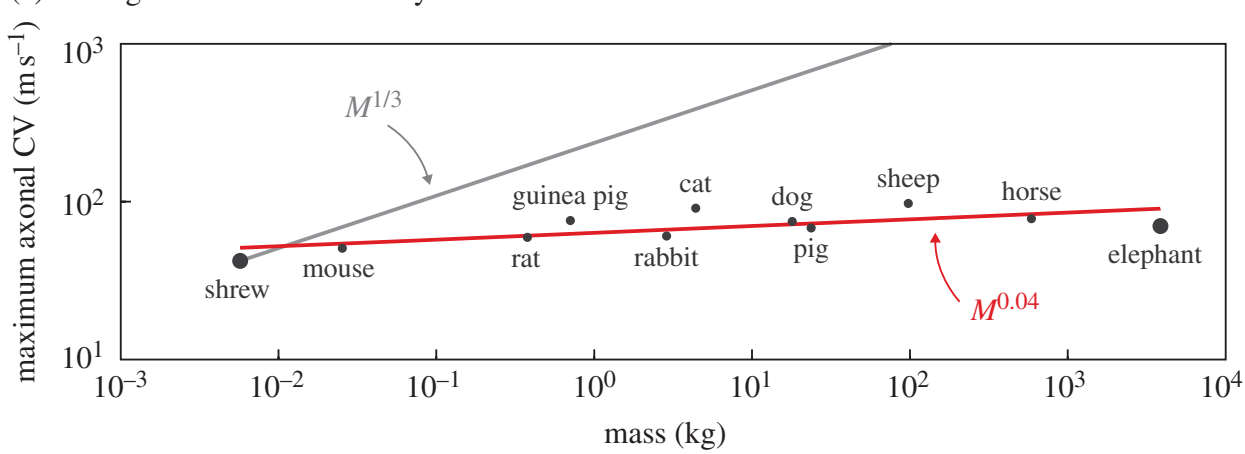

Figure 2. Maximum axonal conduction velocity $(\mathrm{CV})$. (a) We determined maximum axonal CV in each shrew by stimulating electrically at two points on the sciatic nerve (lightning bolt symbols) and recording the evoked electrical potentials in the medial gastrocnemius muscle (green and blue time-series lines). The distance $\Delta d$ between stimulation electrodes was divided by the time difference $\Delta t$ of muscle activity onset to determine CV. (b) We determined maximum axonal CV in the elephant by stimulating the sciatic nerve electrically (lightning bolt symbol) and the Achilles tendon mechanically (reflex hammer symbol). The evoked electrical potentials in the medial gastrocnemius were recorded, and CV determined as in figure $2 a$. (c) Relationship between maximum axonal CV and body mass for 11 species of animals. Apart from elephant and shrew values, data points are averages of several studies (table $\mathrm{S} 1$ in the electronic supplementary material). Geometric similarity in axon anatomy predicts that $\mathrm{CV}$ will increase with mass (grey line), but our results show that it is in fact relatively constant for all animals (red line).

program (Matlab 2007a, The MathWorks, Inc., Natick, MA, USA) to give mosaics showing the whole nerve.

\section{RESULTS AND DISCUSSION}

We found that maximum axonal $\mathrm{CV}$ and axon diameter did not change with animal size to the extent required to maintain sensorimotor responsiveness. Maximum shrew axonal CV was $42 \pm 6 \mathrm{~m} \mathrm{~s}^{-1}$ (mean \pm standard deviation) and maximum elephant axonal $\mathrm{CV}$ was $70 \mathrm{~m} \mathrm{~s}^{-1}$, which when combined with literature data and fit using firstorder least-squares regression yielded a relationship between mass $M$ and axonal $\mathrm{CV}$ of $\mathrm{CV} \propto M^{0.04 \pm 0.03}$ (exponent shown as mean $\pm 95 \%$ confidence interval) (figure $2 c$ ). The very small scaling exponent indicates that maximum axonal $\mathrm{CV}$ is nearly independent of animal size and significantly different than that predicted by geometric similarity (figure $2 c$ ). Similarly, our initial measurements of axon size indicate that the largest elephant axons $(15 \mu \mathrm{m})$ are only about twice the diameter of the largest shrew axons $(7 \mu \mathrm{m})$ (figure 3). Across a 100fold increase in leg length, maximum axonal $\mathrm{CV}$ less than doubled resulting in elephants experiencing conduction delays more than 50 times longer than those of shrews.

The time it takes to respond to stimuli will be much longer in larger animals. For example, consider a locomoting animal whose foot is perturbed just as it contacts the ground. Absolute delay will increase approximately with body mass to the $1 / 3$ power because $C V$ is nearly independent of size and leg length increases in a nearly geometrically similar manner (Alexander 1979) (electronic supplementary material). This long absolute delay is partially, but not completely, offset by the longer absolute time that large animals have available to respond to stimuli owing to their movement patterns. For example, at equivalent speeds, stance duration increases with size - an advantage if an animal needs to respond to the perturbation while the foot is still in contact with the ground. However, stance duration increases only with about mass to the $1 / 6$ power (Heglund et al. 1974; Biewener 1983). As a consequence, conduction delay expressed as a fraction of the stance phase duration increases approximately with mass to the $1 / 6$ power (figure 4; electronic supplementary material). 


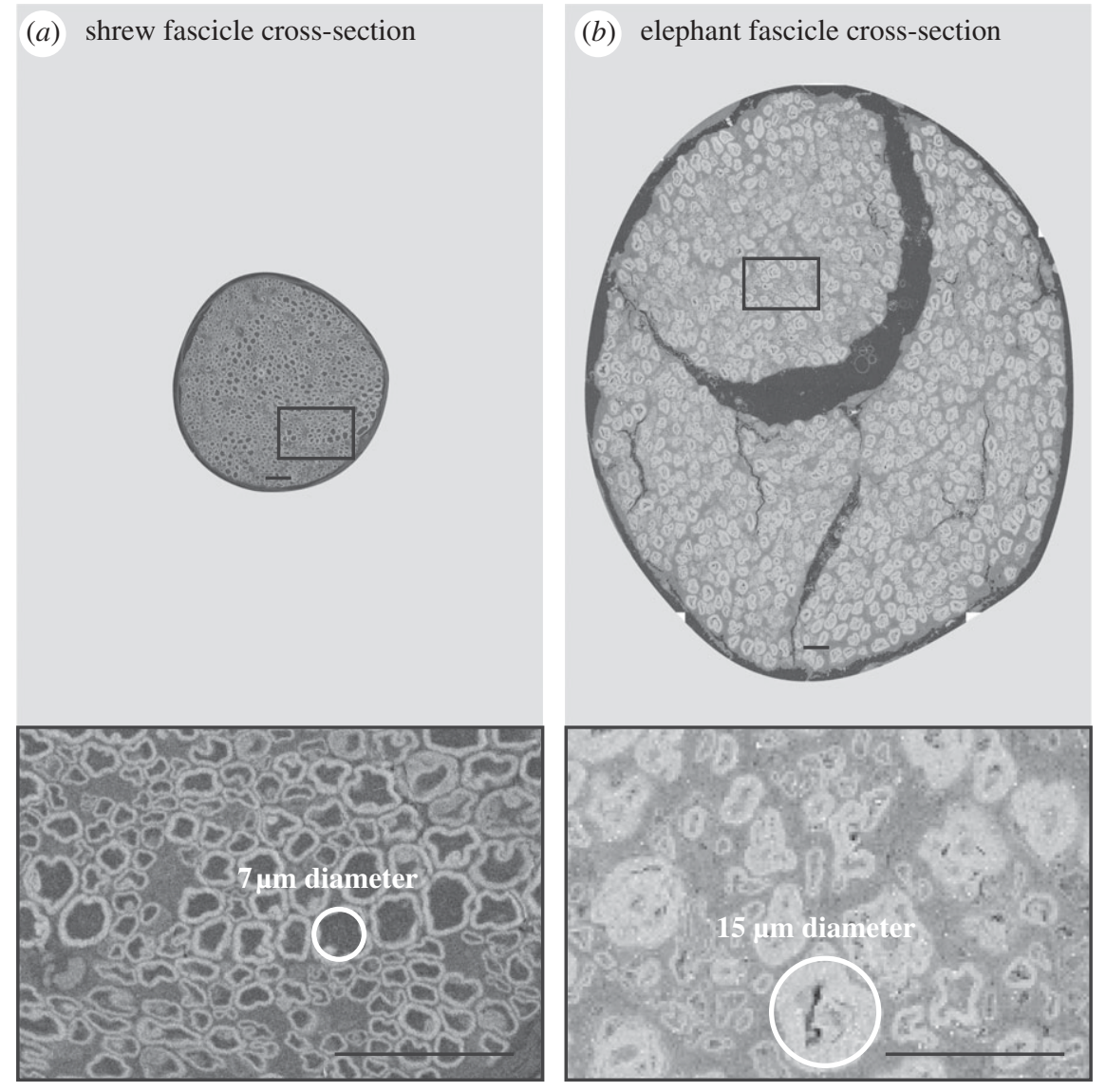

Figure 3. Shrew and elephant nerve cross sections demonstrate that the largest elephant axons are only about twice the diameter of the largest shrew axons. Images in the top row are mosaics of many (10 shrew, 41 elephant) scanning electron microscopy (SEM) images, with the bottom row of images showing single SEM images (electronic supplementary material). Shrew and elephant images are shown at the same scale, with all scale bars $25 \mu \mathrm{m}$ in length. (a) One fascicle, of about 6 , from a shrew sciatic nerve. The largest shrew axons have an outside diameter of approximately $7 \mu \mathrm{m}$ as illustrated by the overlaid white circle. (b) One fascicle, of about 110, from an elephant sciatic nerve. The largest elephant axons have an outside diameter of approximately $15 \mu \mathrm{m}$ - only twice the diameter of those in the shrew.

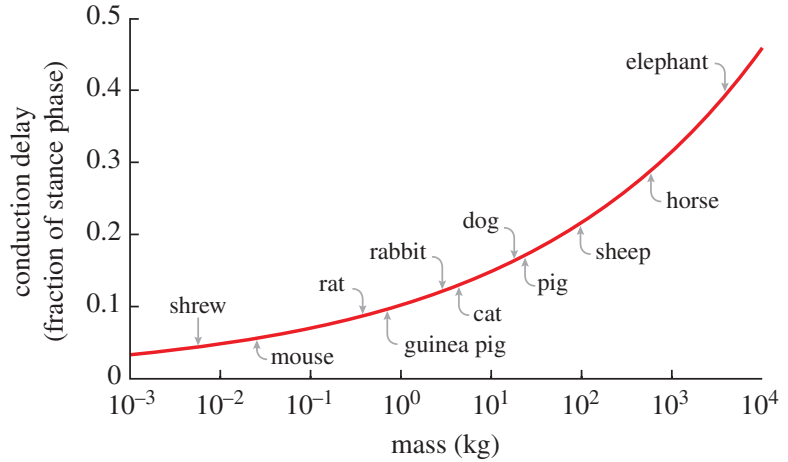

Figure 4. Theoretical scaling of conduction delay. Conduction delay, when represented as a fraction of stance phase at the trot-gallop transition, scales with $M^{0.16}$ (electronic supplementary material). That is, conduction delay takes up a larger fraction of stance phase as animal mass increases. The arrows represent theoretical conduction delay fractions for each of the 11 species considered in figure $2 c$.

The contribution of conduction delay to the time required to respond to the hypothetical perturbation to the foot when running at equivalent speeds would increase from 4 per cent of the stance phase in the shrew to 40 per cent of the stance phase in the elephant. Additional sources of delay, including sensing, transmission and force generation only add to the total delay.
Large animals may cope with these relatively long delays by simply moving slowly, explaining at least in part the low maximum speeds of large mammals (Garland 1983; Hutchinson et al. 2006) and providing further evidence for the idea that dinosaurs could not be both massive and agile (Hutchinson \& Garcia 2002).

Simple feedback control is less effective when time delays are long. By the time sensory signals are conducted from the periphery to the central nervous system, the information they contain about the state of the body is no longer current. Similarly, by the time motor commands are conducted to the periphery, they may be inappropriate for the new body state. The central nervous system can compensate for these delays using an internal model of the body's dynamics that takes delayed and incomplete sensory information and predicts the best future motor response (Wolpert \& Ghahramani 2000; Biewener \& Daley 2007). As delays increase in larger animals, they may increasingly depend upon sensorimotor prediction to maintain sensorimotor performance.

All procedures were approved by the SFU Animal Care Committee.

The authors thank BBSRC, CIHR, MSFHR and NSERC for funding this project. We also thank the Edmonton Valley Zoo, the Colchester Zoo, Zoltan Kenwell, Alejandro Ley and Sharon Warner for assistance with the elephant 
experiments, Orin Mock for providing the shrews, SFU Animal Care Services for assistance with the shrew experiments, and Winnie Enns for assistance with the shrew experiments and nerve processing. In addition, we thank Arthur Prochazka for his insights into data analysis, Kevin Middleton for assistance with the phylogenetically independent contrasts analysis, and Rodger Kram and Art Kuo for feedback on manuscript drafts.

\section{REFERENCES}

Alexander, R. M. 1979 Allometry of the limb bones of mammals from shrews (Sorex) to elephant (Loxodonta). f. Zool. Lond. 189, 305-314. (doi:10.1111/j.1469-7998. 1979.tb03964.x)

Alexander, R. M. 2002 Stability and manoeuvrability of terrestrial vertebrates. Integr. Comp. Biol. 42, 158-164. (doi:10.1093/icb/42.1.158)

Arbuthnott, E. R., Boyd, I. A. \& Kalu, K. U. 1980 Ultrastructural dimensions of myelinated peripheral nerve fibres in the cat and their relation to conduction velocity. F. Physiol. 308, 125-157.

Biewener, A. A. 1983 Allometry of quadrupedal locomotion: the scaling of duty factor, bone curvature and limb orientation to body size. F. Exp. Biol. 105, $147-171$.

Biewener, A. A. \& Daley, M. A. 2007 Unsteady locomotion: integrating muscle function with whole body dynamics and neuromuscular control. f. Exp. Biol. 210, 2949-2960. (doi:10.1242/jeb.005801)

Boyd, I. A. \& Kalu, K. U. 1979 Scaling factor relating conduction-velocity and diameter for myelinated afferent nerve-fibers in the cat hind-limb. F. Physiol. 289, $277-297$.

Enoka, R. M. 1995 Morphological features and activation patterns of motor units. F. Clin. Neurophysiol. 12, $538-559$.

Garland, T. J. 1983 The relation between maximal running speed and body mass in terrestrial mammals. F. Zool. Lond. 199, 157-170. (doi:10.1111/j.1469-7998.1983. tb02087.x)
Gasser, H. S. \& Grundfest, H. 1939 Axon diameters in relation to the spike dimensions and the conduction velocity in mammalian a fibers. Am. F. Physiol. 127, 393-414.

Heglund, N. C., Taylor, C. R. \& McMahon, T. A. 1974 Scaling stride frequency and gait to animal size: mice to horses. Science 186, 1112-1113. (doi:10.1126/science. 186.4169.1112)

Hoffmeister, B., Janig, W. \& Lisney, S. J. 1991 A proposed relationship between circumference and conduction velocity of unmyelinated axons from normal and regenerated cat hindlimb cutaneous nerves. Neuroscience 42, 603-611. (doi:10.1016/0306-4522(91)90402-A)

Hursh, J. B. 1939 Conduction velocity and diameter of nerve fibers. Am. F. Physiol. 127, 131-139.

Hutchinson, J. R. \& Garcia, M. 2002 Tyrannosaurus was not a fast runner. Nature 415, 1018-1021. (doi:10. 1038/4151018a)

Hutchinson, J. R., Schwerda, D., Famini, D. J., Dale, R. H., Fischer, M. S. \& Kram, R. 2006 The locomotor kinematics of Asian and African elephants: changes with speed and size. f. Exp. Biol. 209, 3812-3827. (doi:10.1242/jeb.02443)

Matthews, P. 1972 Mammalian muscle receptors and their central actions. London, UK: Edward Arnold (Publishers) Ltd.

McMahon, T. A. \& Bonner, J. T. 1983 On size and life. New York, NY: Scientific American Library. Distributed by W.H. Freeman.

Miller, C. E., Basu, C., Fritsch, G., Hildebrandt, T. \& Hutchinson, J. R. 2008 Ontogenetic scaling of foot musculoskeletal anatomy in elephants. F. R. Soc. Interface 5, 465-475. (doi:10.1098/rsif.2007.1220)

Misiaszek, J. E. 2003 The H-reflex as a tool in neurophysiology: its limitations and uses in understanding nervous system function. Muscle Nerve 28, 144-160. (doi:10.1002/mus.10372)

Rushton, W. A. 1951 A theory of the effects of fibre size in medullated nerve. F. Physiol. 115, 101-122.

Wolpert, D. M. \& Ghahramani, Z. 2000 Computational principles of movement neuroscience. Nat. Neurosci. 3(Suppl.), 1212-1217. 